Epidemiology and Infection

cambridge.org/hyg

\section{Original Paper}

Cite this article: Adekunle $\mathrm{Al}$, Adegboye OA, Gayawan E, McBryde ES (2020). Is Nigeria really on top of COVID-19? Message from effective reproduction number. Epidemiology and Infection 148, e166, 1-7. https://doi.org/ $10.1017 /$ S0950268820001740

Received: 16 May 2020

Revised: 24 July 2020

Accepted: 31 July 2020

\section{Key words:}

Basic reproduction number; COVID-19; effective reproductive number; imported cases; Nigeria

Author for correspondence:

A. I. Adekunle,

E-mail: kunle102@yahoo.com

\title{
Is Nigeria really on top of COVID-19? Message from effective reproduction number
}

A. I. Adekunle ${ }^{1,2}$ (D), O. A. Adegboye ${ }^{1}$ (D) E. Gayawan ${ }^{3}$ (D) and E. S. McBryde ${ }^{1}$

${ }^{1}$ Australia Institute of Tropical Health and Medicine, James Cook University, Townsville, Australia; ${ }^{2}$ Decision and Modelling Science, Victoria University, Melbourne, Australia and ${ }^{3}$ Biostatistics and Spatial Statistics Research Group, Department of Statistics, Federal University of Technology, Akure, Nigeria

\section{Abstract}

Following the importation of coronavirus disease (COVID-19) into Nigeria on 27 February 2020 and then the outbreak, the question is: How do we anticipate the progression of the ongoing epidemic following all the intervention measures put in place? This kind of question is appropriate for public health responses and it will depend on the early estimates of the key epidemiological parameters of the virus in a defined population.

In this study, we combined a likelihood-based method using a Bayesian framework and compartmental model of the epidemic of COVID-19 in Nigeria to estimate the effective reproduction number $(R(t))$ and basic reproduction number $\left(R_{0}\right)$ - this also enables us to estimate the initial daily transmission rate $\left(\beta_{0}\right)$. We further estimate the reported fraction of symptomatic cases. The models are applied to the NCDC data on COVID-19 symptomatic and death cases from 27 February 2020 and 7 May 2020.

In this period, the effective reproduction number is estimated with a minimum value of 0.18 and a maximum value of 2.29. Most importantly, the $R(t)$ is strictly greater than one from 13 April till 7 May 2020. The $R_{0}$ is estimated to be 2.42 with credible interval: (2.37-2.47). Comparing this with the $R(t)$ shows that control measures are working but not effective enough to keep $R(t)$ below 1 . Also, the estimated fraction of reported symptomatic cases is between 10 and $50 \%$.

Our analysis has shown evidence that the existing control measures are not enough to end the epidemic and more stringent measures are needed.
(C) The Author(s), 2020. Published by Cambridge University Press. This is an Open Access article, distributed under the terms of the Creative Commons Attribution licence (http://creativecommons.org/licenses/by/4.0/), which permits unrestricted re-use, distribution, and reproduction in any medium, provided the original work is properly cited.

\section{CAMBRIDGE} UNIVERSITY PRESS

\section{Introduction}

The emerging severe acute respiratory syndrome coronavirus 2 (SARS-CoV-2) causing COVID-19 is still causing havoc globally. The outbreak was declared a pandemic by the World Health Organisation (WHO) on 11 March 2020. As of 2 May 2020, over 3.5 million people were confirmed to be infected with this respiratory disease, and about a quarter of a million deaths have been reported globally [1]. Many countries have put intervention measures in place to contain the virus and progress has been reported in many countries [2]. Despite the reported successes, the pandemic is far from being over at a global scale.

Nigeria can be said to be in the early stage of COVID-19 dynamics [3]. The first case of COVID-19 importation to the country was reported on 27 February 2020 and since then, there have been many more local and imported cases [4]. Following this outbreak, the Nigerian government placed a ban on international flights to prevent seeding from other affected countries and introduce lockdown and social distancing to reduce local transmissions. However, the effectiveness of these measures in Nigeria is yet to be determined.

There are many challenges facing the Nigerian Government responses to COVID-19 epidemics. As typical to the African continent, Nigeria is already burdened by other diseases such malaria, tuberculosis, yellow fever and others [5]. Thus, combating COVID-19 in the presence of these diseases requires a well-planned strategy [6]. Nigeria lacks the capacity to provide adequate care for patients infected with COVID-19, perform contact tracing and conduct testing $[6,7]$. Treatment of complicated COVID-19 infection requires ventilators, uninterrupted power supply and oxygen. These are challenges in Nigeria coupled with poor infrastructure and building conducive isolation centres for mandatory quarantine of suspected and infected cases. In addition to these challenges, the northeastern states of Nigeria are conflict affected and it will be very difficult to implement epidemic control in conflict-affected regions [8]. Nonetheless, it is important to determine whether progress has been made or not in the fight against COVID-19.

An important parameter that is crucial for understanding the dynamics of any infectious disease is the effective reproduction number $(R(t))$, which must be reevaluated as the pandemic progresses to determine whether or not there is progress in containing the situation [9]. The $R(t)$ is related to the basic reproduction number $\left(R_{0}\right)$, which is defined as the average 
number of secondary cases per typical infected case in a fully susceptible population before interventions have been put in place. As the epidemic progresses, the susceptible pool decreases and hence, $R(t)=R_{0} x$, where $x$ is the fraction of the population that is susceptible to infection. $R(t)$ is also influenced by control measures to mitigate or eradicate infectious diseases. These quantities $R(t)$ and $R_{0}$, can help characterise the growth rate of an epidemic, inform recommendation for control measures and determine the impact of control measures. When $R(t)<1$, the epidemic can no longer be maintained and will begin to decline. Otherwise, additional measures are needed or else the epidemics will continue to grow.

To understand the dynamics of COVID-19 in Nigeria, we used the likelihood-based model that was previously introduced for the Ebola pandemic [10] and adopted for COVID-19 [11] to estimate $R(t)$ in a Bayesian framework. The approach adjusts for the contribution of imported cases and report fraction of the transmission process.

\section{Material and methods}

\section{Data sources}

Daily counts of confirmed COVID-19 cases were extracted from publicly available data provided by the Nigeria Centre for Disease Control (NCDC) [4]. The NCDC daily situation reports include the number of local and imported cases. From 16 April 2020, all reported cases were classified as local cases by NCDC. This suggests that the travel ban imposed by the Nigerian Government on 20 March 2020 was effective. However, due to border porosity in Africa [12], there is a high likelihood that some of the reported cases after 20 March 2020 were imported cases or some imported COVID-19 cases were unreported.

\section{Effective reproduction number estimation}

We adopted a likelihood-based approach that allows for the evaluation of the contributions of imported cases of COVID-19 [10, 11, 13]. The locally acquired infections $(L(t))$ are assumed to be Poisson distributed with rate given as

$$
\lambda(t)=\left(R(t) \sum_{s=1}^{t} \psi(s) C(t-s)\right)
$$

where $C(t)$ is the number of daily new cases (locally acquired and importations) on day $t=0, \ldots, T . \psi(s)$ is the distribution of the serial interval. We assumed that $\psi(s)$ is lognormal distributed with a mean of 4.7 days and a standard deviation of 2.9 days [14]. The likelihood $\mathcal{L}$ of the observed times series of cases from day 1 to day $T$ conditional on $C(0)$ is thus given by

$$
\mathcal{L}=\prod_{t=1}^{T} P(L(t) ; \lambda(t))
$$

where $P$ is the probability mass function of a Poisson distribution. The effective reproduction is estimated in a Bayesian framework using the Metropolis-Hasting Markov chain Monte Carlo (MCMC) method for sampling from the prior distribution of $R$ $(t)$. We assumed a non-informative prior, uniform $(0,5)$ for $R$ $(t)$ and performed 1000000 iterations with a burn-in of $10 \%$.
The effective reproduction number shows how effective are the intervention strategies. The interventions put in place in Nigeria include disease surveillance to capture importations and local transmission, travel ban, contact tracing, isolation, quarantine and social distancing [4]. With a score of approximately $40 \%$ on the WHO Joint External Evaluation (JEE) mission report for Nigeria's capacity to prevent, detect and rapidly respond to public health risk [15], these metrics do not favour Nigeria's COVID-19 outcomes. Therefore, to obtain the proportion of symptomatic under-reported in the daily COVID-19 cases, we applied the delay-adjusted cases fatality ratio [16]. Detail explanation of the model can be found in Riou et al. [16].

\section{Assumptions and sensitivity analysis}

Previous work has shown that apporximately 50\% of COVID-19 cases are asymptomatic [17]. Hence, we doubled the adjusted reported cases prior to estimating $R(t)$. We used the following different assumptions about cases detection to test the sensitivity of the estimation of the effective reproduction number to assumptions.

(1) All importations are equally likely to cause infection as local cases.

(2) $40 \%$ of all importations contribute to transmission. Here, Im $(t)=40 \operatorname{Im}(t) / 100$. This is saying that the imported cases contributed less to the transmission and $\operatorname{Im}(t)$ is the number of imported cases per day.

In the case in which we assume $40 \%$ reporting proportion, we interpreted it as reporting probability to adjust for the underreporting in COVID-19 cases as

$$
\text { report } \sim \operatorname{Binomial}(H(t), p)
$$

here, $H(t)$ is the adjusted reported number of cases and $p$ is the reporting proportion.

\section{Modelling the dynamics of COVID-19}

To forecast the number of COVID-19 cases, we estimate the basic reproduction number $\left(R_{0}\right)$ instead of $R(t)$ using a compartmental model which was fitted in a Bayesian framework to the cumulative reported cases and deaths data. The compartmental model is a general SEIR-type model ( $\mathrm{S}$ - susceptible, E - exposed, I - infectious and $\mathrm{R}$ - recovered). In the model, we divide the population into 10 compartments (see Table 1). The deterministic form of the model is given below

$$
\begin{gathered}
\frac{\mathrm{d} S}{\mathrm{~d} t}=-\frac{\left(\beta(t)\left(I_{1}+2 I_{2}+I_{3}\right)\right) S}{N} \\
\frac{\mathrm{d} E_{1}}{\mathrm{~d} t}=\frac{\left(\beta(t)\left(I_{1}+2 I_{2}+I_{3}\right)\right) S}{N}-\sigma_{1} E_{1} \\
\frac{\mathrm{d} E_{2}}{\mathrm{~d} t}=\sigma_{1} E_{1}-\sigma_{2} E_{2} \\
\frac{\mathrm{d} I_{1}}{\mathrm{~d} t}=\rho_{1} \sigma_{2} E_{2}-\gamma_{1} I_{1}
\end{gathered}
$$




$$
\begin{gathered}
\frac{\mathrm{d} I_{2}}{\mathrm{~d} t}=k_{1} C+\gamma_{1} I_{1}-\gamma_{2} I_{2} \\
\frac{\mathrm{d} I_{3}}{\mathrm{~d} t}=\left(1-\rho_{1}\right) \sigma_{2} E_{2}-\gamma_{3} I_{3} \\
\frac{\mathrm{d} R}{\mathrm{~d} t}=\rho_{2} \gamma_{2} I_{2}+\gamma_{3} I_{3} \\
\frac{\mathrm{d} D}{\mathrm{~d} t}=\left(1-\rho_{2}\right) \gamma_{2} I_{2} \\
\frac{\mathrm{d} C}{\mathrm{~d} t}=k_{1} C \\
\frac{\mathrm{d} H}{\mathrm{~d} t}=\rho_{3}\left(k_{1} C+\gamma_{1} I_{1}\right)
\end{gathered}
$$

where $D$ is the death compartment, $C$ is the importation compartment and $H$ is the hospitalised compartment. The model is closed population model with no births or non-COVID-19 mortality.

The basic reproduction number estimated using the nextgeneration method [18] is given as

$$
R_{0}=\beta\left(\frac{\rho_{1}}{\gamma_{1}}+\frac{2 \rho_{1}}{\gamma_{2}}+\frac{\left(1-\rho_{1}\right)}{\gamma_{3}}\right)
$$

The model parameters and estimates are described in Table 2. The analysis was performed in R [19] and the codes for the estimation can be download from https://github.com/Shina-GitHub/ COVID-19_Nig.

\section{Results}

\section{Epidemic data}

Table 3 presents the summary statistics of the burden of COVID-19 epidemics in Nigeria from 27 February to 7 May 2020. During this period, there was a total of 3322 local cases, 210 imported cases and 107 deaths. The death counts bring the case-fatality rate to $3.03 \%$, a reduction in what was reported for the first 45 days of the epidemics [3].

\section{Baseline effective reproduction number}

We first estimate the effective reproduction number assuming there was no reporting delay (reporting proportion was 100\%) and all the imported cases were as infectious as the locally acquired cases. The first local case (a contact of the index case) was reported on 9 March 2020, about 11 days after the importation of the first oversea case. Figure $1 a, b$ shows the baseline effective reproductive number and the model fit. The figure reveals that the effective reproduction number was below 1 for the first three weeks since the introduction and increased above the threshold of 1 thereafter. This implies that the mitigation strategy is not optimally controlling the disease as $R(t)$ is gradually increasing. The minimal value estimation of $R(t)$ was 0.18 and the maximal value was 2.29 . The corresponding model fit
Table 1. State variable description

\begin{tabular}{ll}
\hline Variable & Description \\
\hline$S$ & Susceptible individuals \\
\hline$E_{1}$ & Exposed individuals \\
\hline$E_{2}$ & $\begin{array}{l}\text { Individuals in the pre-symptomatic transmission stage } \\
\text { during the incubation period }\end{array}$ \\
\hline$I_{1}$ & $\begin{array}{l}\text { Individuals that are between onset symptoms and } \\
\text { presentation to health care stage }\end{array}$ \\
\hline$I_{2}$ & Individuals with confirmed COVID-19 case and infections \\
\hline$R$ & Individuals asymptomatic \\
\hline$D$ & Recovered class \\
\hline$C$ & Individuals who died from COVID-19 \\
\hline$H$ & Cumulative CoviD-19 importations \\
\hline
\end{tabular}

and bar graphs of the reported local and imported cases are shown in Figure $1 b$.

We further estimate $R(t)$ by assuming that $40 \%$ of the imported cases contributed to transmission (Fig. 2a). The $R(t)$ estimates showed the same pattern as the baseline model except that $R(t)$ was further above 1 in the first three weeks since the first local case was reported. The minimal value of $R(t)$ was 0.22 and maximal value of 3.14. The higher value in the peak of $R(t)$ implies that the burden of the COVID-19 is more than imagined.

\section{Effects of symptomatic reporting proportion on effective reproduction number}

We adopted the model developed by Russell et al. [17] to estimate the COVID-19 symptomatic reporting proportion for Nigeria (Fig. 2b). The results show that the under-reporting in the reported cases ranged from $10 \%$ to $50 \%$ in the two months of the outbreak. The estimate is quite large suggesting that many potential infected cases in the community are not being reported. Again, using equation (3), we estimated $R(t)$ to determine the effect of underreporting. We first use equation (3) to adjust for under-reporting and then double the number to adjust for asymptomatic infection [16]. The estimated $R(t)$ for $10 \%$ reporting proportion was similar to the estimates without adjusting for underreporting. However, the initial $R(t)$ was 4.78 (Fig. $3 a$ ). This implies that the initial rate of infection was high early in the outbreak and the mitigating controls were able to reduce the burden. Although, when we assumed relative infectiousness, the epidemic was relatively suppressed until first week of May 2020 . With the estimates of $R(t)$ above 1 in the first week of May implies that the disease is increasing again. Similar conclusion can be reached for $50 \%$ reporting proportion (Fig. $3 b$ ).

\section{Model dynamics and scenario analysis}

Using the compartment model [4], we estimated the basic reproduction number $R_{0}$ and forecasted the cumulative number of COVID-19 cases in Nigeria for 3 weeks ahead assuming that the present dynamics is maintained. The estimated $R_{0}$ was $2.42(95 \%$ credible interval: 2.37-2.47). This estimate was similar to the $R_{0}$ estimated for Wuhan during the early dynamics of COVID-19 in 
Table 2. Parameter descriptions

\begin{tabular}{|c|c|c|}
\hline Parameter & Description & Values (references) \\
\hline $\boldsymbol{\beta}(\boldsymbol{t})=\boldsymbol{\beta}_{0}$ & Transmission rate & $0.315\left(95 \% \mathrm{Cl}^{\star} 0.309-0.321\right)$ \\
\hline$\sigma_{1}$ & First-stage incubation rate & $0.3125[20$ \\
\hline$\sigma_{2}$ & Second-stage incubation rate & $0.5[20]$ \\
\hline$\rho_{1}$ & Proportion progressing from $E_{2}$ to $I_{1}$ & $0.5[16,20]$ \\
\hline$\rho_{2}$ & Proportion that recovered & $0.944\left(95 \% \mathrm{Cl}^{\star} 0.940-0.948\right)$ \\
\hline$\rho_{3}$ & Relative reporting parameter & $0.982\left(95 \% \mathrm{Cl}^{\star} 0.934-1\right)$ \\
\hline$\gamma_{1}$ & First stage of recovery & $0.5[20]$ \\
\hline$\gamma_{2}$ & Second stage of recovery & $0.176[20]$ \\
\hline$\gamma_{3}$ & First stage of recovery & $0.5[20]$ \\
\hline $\boldsymbol{k}_{1}$ & Importation rate & $0.091\left(95 \% \mathrm{Cl}^{\star} 0.086-0.095\right)$ \\
\hline$S(0)$ & Initial susceptible population & $1.98 \times 10^{8}[21]$ \\
\hline$C(0)$ & Initial cumulative imported cases & $2[22]$ \\
\hline$H(0)$ & Initial symptomatic case local & $1[22]$ \\
\hline$I_{2}(0)$ & Initial symptomatic cases total & $I_{2}(0)=C(0)+H(0)$ \\
\hline
\end{tabular}

${ }^{\star} \mathrm{Cl}$-credible interval, estimated parameters are shown in bold. The state variables not shown in the table are set to zero.

Table 3. Descriptive statistics of the epidemic data of COVID-19 in Nigeria between 27 February and 7 May

\begin{tabular}{lccccccc}
\hline Variable & Mean & SD & Minimum & Q1 & Median & Q3 $^{*}$ & Maximum \\
\hline Local cases & 70.68 & 89.34 & 0.00 & 3.50 & 22.00 & 111.00 & 381.00 \\
\hline Imported cases & 7.78 & 6.39 & 1.00 & 2.50 & 7.00 & 10.00 & 30.00 \\
\hline Death cases & 0.87 & 2.29 & 0 & 0 & 0 & 17 \\
\hline
\end{tabular}

Q1* - first quartile, $\mathrm{Q}^{\star}$ - third quartile.
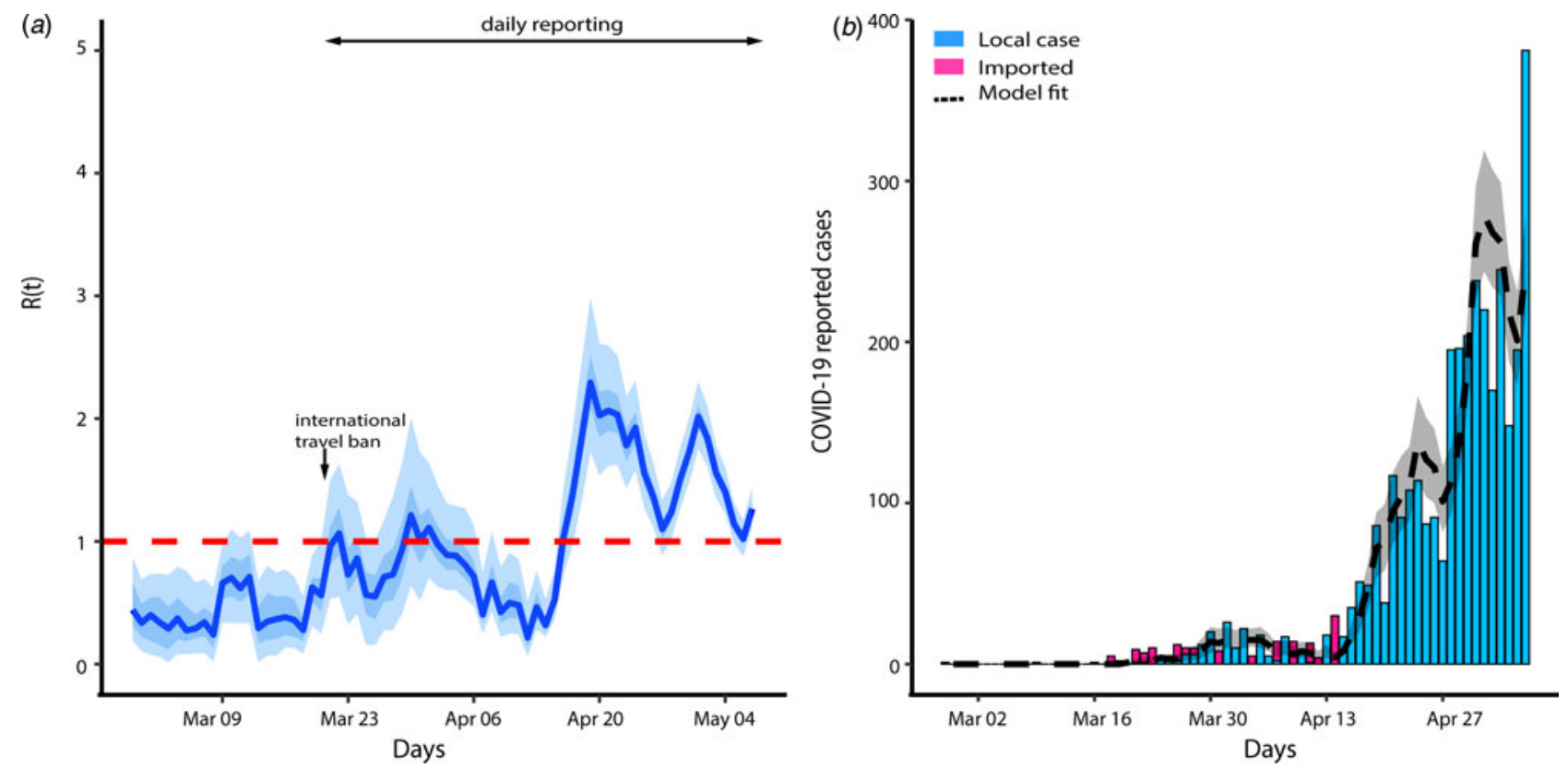

Fig. 1. (a) Baseline effective reproduction number. The light and dark blue colour show $95 \%$ and $50 \%$ credible interval, respectively, and the solid line is the median estimate. $(b) \boldsymbol{R}(\boldsymbol{t})$ model fit. The black dashed line is the median estimation of the local cases and the grey area shows a $95 \%$ credible interval of the estimated local cases. 
(a)

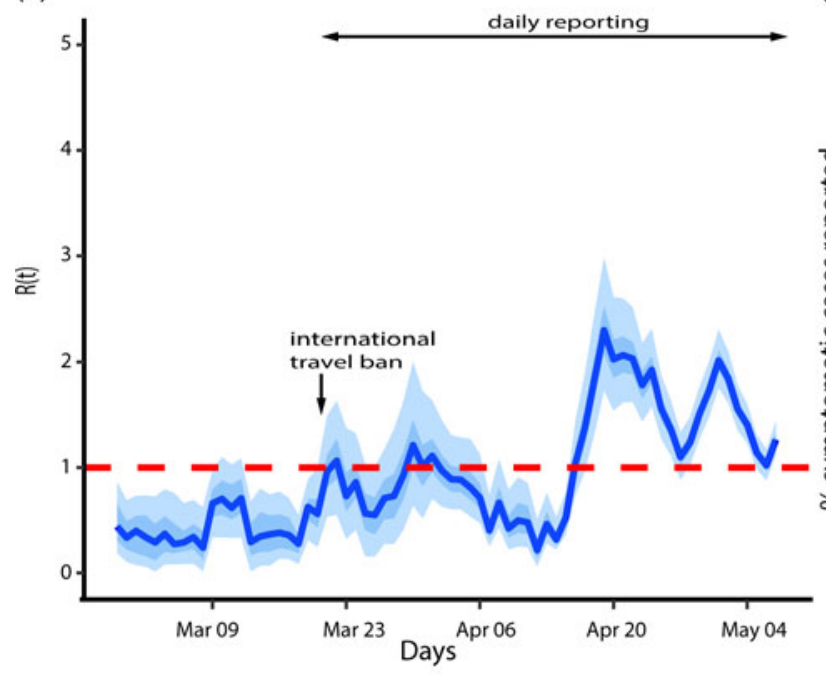

(b)

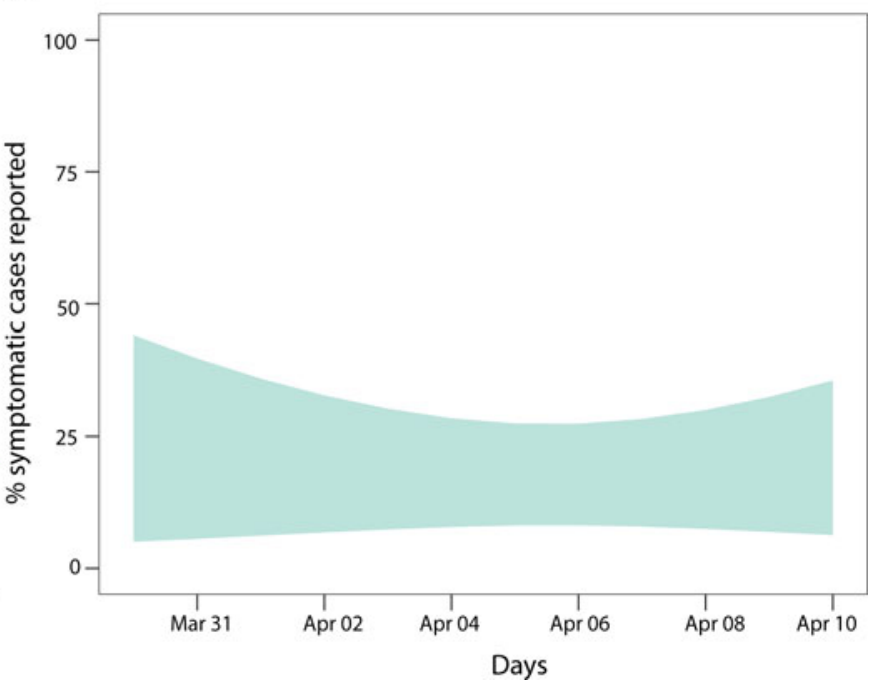

Fig. 2. (a) Adjusted effective reproduction number for Nigeria COVID-19 epidemics based on $40 \%$ of imported cases contributing to transmission, (b) COVID-19 symptomatic reporting measure.

(a)

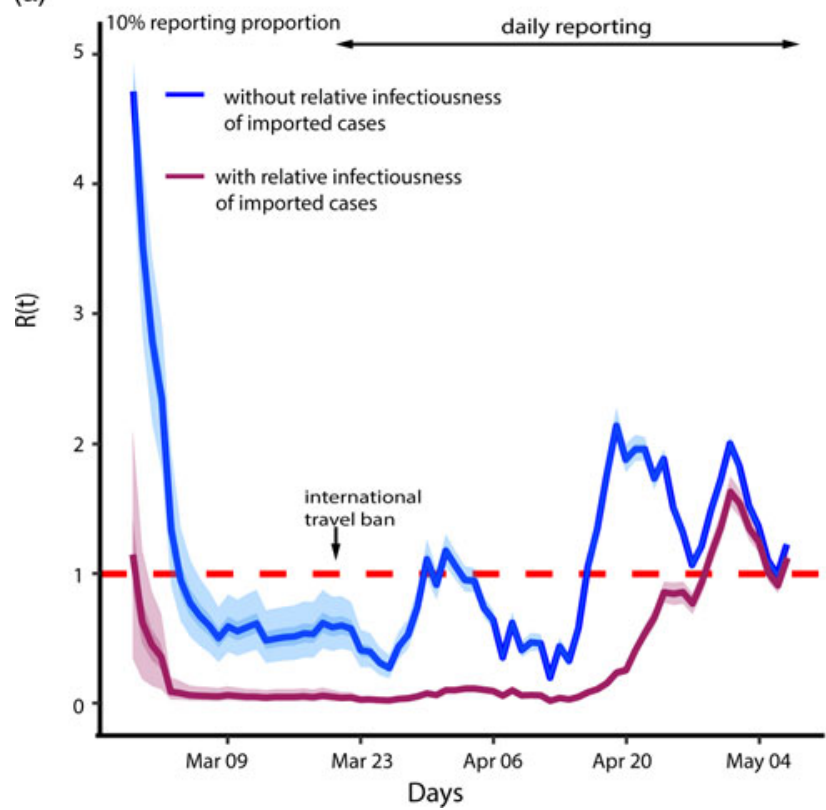

(b)

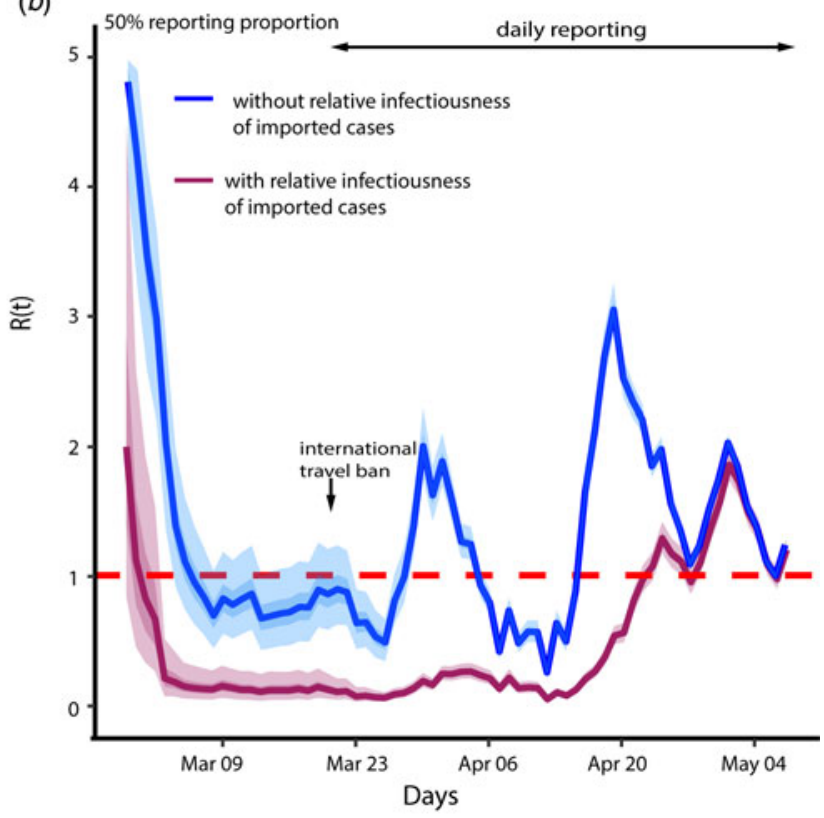

Fig. 3. Effect of reporting proportion on effective reproduction number. (a) 10\% reporting proportion, (b) 50\% reporting proportion.

China $[23,24]$. The 3 -week forecasts for the cumulative reported number of cases and deaths are shown in Figure $4 a, b$, respectively. Our model predicted a $95 \%$ credible interval of 58890 and 69739 for cumulative reported cases by 28 May 2020 and between 1953 and 2238 for cumulative reported death cases.

\section{Discussion}

In this study, we estimated the effective reproduction number and basic reproduction for COVID-19 epidemic in Nigeria for the period between 27 February 2020 and 7 May 2020. Our modelling results showed that the trajectory of COVID-19 pandemic in Nigeria is far from over as the effective reproduction number is above the threshold of 1 and increasing. This indicates that the mitigation measures put in place have not been effective enough and efforts must be concentrated on ensuring that the existing measures are improved and additional measures that synergise the existing ones can be introduced. The estimated basic reproduction number of COVID-19 for Nigeria is 2.63. This is similar to what was estimated for China in the early phase of the pandemic [23]. With this estimate, it is clear that the mitigation measures are not able to control the disease to the level that will flatten the curve or quash it. We further determine the fraction of symptomatic proportions reported using existing work. This clearly shows that the burden of COVID-19 in the country may be more than reported. 
(a)

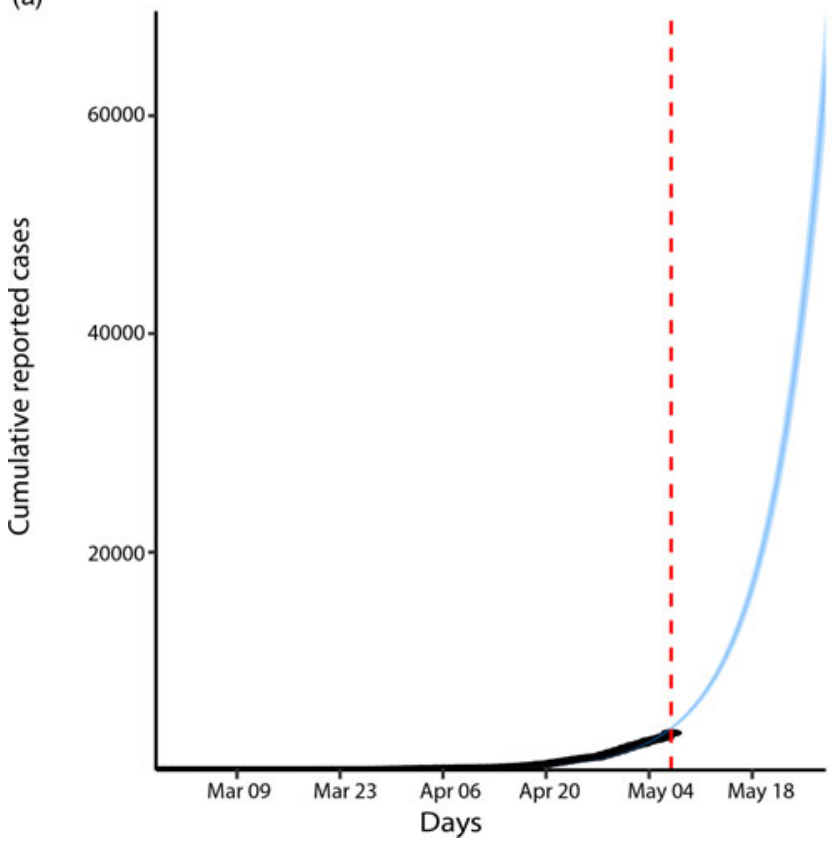

(b)

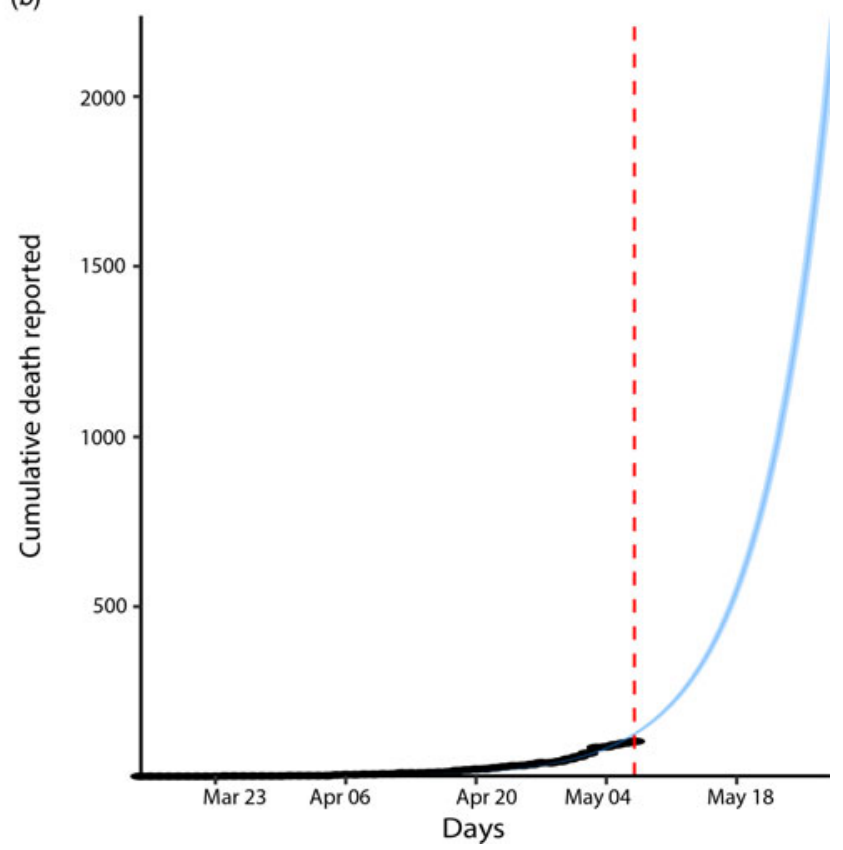

Fig. 4. Model fit and (a) forecast of the cumulative reported cases, (b) COVID-19 deaths.

The results presented in this study should be interpreted as a guild line with the following caveats in mind. First, imported cases are not reported daily. Considering the porosity of Nigerian borders, we expect more imported cases to be coming from other neighbouring African countries via land [12, 25, 26]. Inadequate reporting may affect our estimates as we might have underestimated the burden of the pandemic because more imported cases that have not been counted for probably contributed to the transmission. Under-reporting of local cases also occurs (proportion symptomatic showed that the reporting rate is between $10 \%$ and $50 \%$ ), however, this is less likely to affect our estimate, because underreporting of local cases will have a multiplier effect on both force of infection and new cases, which cancels out for the estimation of the effective reproduction number. The difference between imported and local cases explains why our estimate of effective reproduction in Figure $1 a$ is far different from the one in Figure 3. Second, the role of asymptomatic patients in the dynamic of COVID-19 has not been fully understood $[27,28]$. There is evidence that these group of people contribute to the transmission process [16, 27, 28]. Though we included them in our compartmental model, the likelihood model does not adjust for asymptomatic patients. This may impair the estimates as what we thought as under-reporting may be many asymptomatic patients who are fuelling transmission and cannot be accounted for unless widespread testing and isolation is conducted. Lastly, the serial interval used in our estimation is derived from infector and infectee data for Japanese patients [14]. This may differ in the Nigerian cohort as variability in innate immune response may affect whether a patient shows symptoms or not. Hence, getting the right pool of patients to characterise the serial interval can be challenging [29].

\section{Conclusion}

Information derived when key epidemic parameters are estimated is important for assessing existing control strategies, adopting more effective alternatives and policy. We have combined existing models with our new model to characterise the pandemic of COVID-19 in Nigeria. This study indicates that more control measures are needed to be able to stop this virus in this epidemic setting. We hope our study can guide public health responses in the fight against COVID-19 in Nigeria.

Data availability statement. The data for this study is openly available on http://doi.org/10.5281/zenodo.3970269.

\section{References}

1. WHO (2020) Situation report -103: WHO; [2 May 2020]. Covid-19 situation report]. Available at https://www.who.int/docs/default-source/ coronaviruse/situation-reports/20200502-covid-19-sitrep-103.pdf?sfvrsn= d95e76d8_4.

2. Salzberger B, Glück T and Ehrenstein B (2020) Successful containment of COVID-19: the WHO-Report on the COVID-19 outbreak in China. Springer.

3. Adegboye OA, Adekunle AI and Gayawan E (2020) Early transmission dynamics of novel coronavirus (COVID-19) in Nigeria. International Journal of Environmental Research and Public Health 17, 3054.

4. Nigeria Centre for Disease Control. COVID-19 Outbreak in Nigeria Situation Report. Abuja, Nigeria: NCDC, 2020 Contract No.: 001-032.

5. Mundi I (2019) Nigeria Major infectious diseases: index Mundi; [13 July 2020]. Available at https://www.indexmundi.com/nigeria/major_infectious_ diseases.html.

6. Lucero-Prisno DE, Adebisi YA and Lin X (2020) Current efforts and challenges facing responses to $2019-\mathrm{nCoV}$ in Africa. Global Health Research and Policy 5, 1-3.

7. Forum WE (2020) Why Sub-Saharan Africa needs a unique response to COVID-19: World Economic Forum; [cited 202013 July]. Available at https://www.weforum.org/agenda/2020/03/why-sub-saharan-africa-needsa-unique-response-to-covid-19/.

8. Tijjani SJ and Ma L (2020) Is Nigeria prepared and ready to respond to the COVID-19 pandemic in its conflict-affected northeastern states? International Journal for Equity in Health 19, 1-4.

9. Fraser C et al. (2004) Factors that make an infectious disease outbreak controllable. Proceedings of the National Academy of Sciences 101, 6146-6151. 
10. WHO Ebola Response Team (2014) Ebola virus disease in West Africa the first 9 months of the epidemic and forward projections. New England Journal of Medicine 371, 1481-1495.

11. Zhang J et al. (2020) Evolving epidemiology and transmission dynamics of coronavirus disease 2019 outside Hubei province, China: a descriptive and modelling study. The Lancet Infectious Diseases 20, 793-802.

12. Okumu W (2011) Border management and security in Africa. Concordis Briefing 4.

13. Liu Q-H et al. (2018) Measurability of the epidemic reproduction number in data-driven contact networks. Proceedings of the National Academy of Sciences 115, 12680-5.

14. Nishiura H, Linton NM and Akhmetzhanov AR (2020) Serial interval of novel coronavirus (COVID-19) infections. International Journal of Infectious Diseases.

15. World Health Organization (2019) Joint External Evaluation (JEE) mission reports.

16. Riou J et al. (2020) Adjusted age-specific case fatality ratio during the COVID-19 epidemic in Hubei, China, January and February 2020. medRxiv.

17. Russell TW et al. (2020) Using a delay-adjusted case fatality ratio to estimate under-reporting. Centre for Mathematical Modeling of Infectious Diseases Repository, posted online March 2020;22.

18. Diekmann O, Heesterbeek J and Roberts MG (2010) The construction of next-generation matrices for compartmental epidemic models. Journal of the Royal Society Interface 7, 873-885.

19. Team RC (2013) R: a language and environment for statistical computing, version 3.0. 2. Vienna, Austria: R Foundation for Statistical Computing, 2019.
20. Funk S and Eggo RM. Early dynamics of transmission and control of 2019-nCoV: a mathematical modelling study.

21. Worldometer (2019) Nigeria Population (live): Worldometer; [cited 20208 July 2020]. Available at https://www.worldometers.info/world-population/ nigeria-population/.

22. NCDC (2020) Covid-19 Outbreak in Nigeria, situation report: NCDC; [cited 2020].

23. Maier BF and Brockmann D (2020) Effective containment explains subexponential growth in recent confirmed COVID-19 cases in China. Science.

24. Wu JT, Leung K and Leung GM (2020) Nowcasting and forecasting the potential domestic and international spread of the 2019-nCoV outbreak originating in Wuhan, China: a modelling study. The Lancet 395, 689697.

25. Okunade SK and Ogunnubi O (2019) The African Union protocol on free movement: a panacea to end border porosity? Journal of African Union Studies 8, 73-91.

26. TImes P (2020) COVID-19: 67 Nigerian returnees from Ivory Coast arrive Seme border Abuja: Premium Yimes; [cited 2020].

27. Day M (2020) Covid-19: identifying and isolating asymptomatic people helped eliminate virus in Italian village. BMJ 368, m1165.

28. Bai Y et al. (2020) Presumed asymptomatic carrier transmission of COVID-19. JAMA 323, 1406-1407.

29. Thevarajan I et al. (2020) Breadth of concomitant immune responses prior to patient recovery: a case report of non-severe COVID-19. Nature Medicine 26, 453-455. 